\title{
Identification of Genes Related to Clinicopathological Characteristics and Prognosis of Patients with Colorectal Cancer
}

\author{
Xueren $\mathrm{Gao}^{1}$ and Jiaojiao Yang ${ }^{2}$
}

The aim of this study was to identify genes with clinical significance in colorectal cancer (CRC). Gene expression profiles of 585 CRC tissues and 61 normal colorectal tissues from Gene Expression Omnibus (GEO) and The Cancer Genome Atlas (TCGA) databases were used to identify differentially expressed genes (DEGs) between CRC and normal colorectal tissues. DAVID and KOBAS tools were used to explore Gene Ontology (GO) and KEGG pathways enriched by DEGs, respectively. In addition, TCGA data sets were also used to identify prognostic factors and develop a prognostic prediction model for CRC. A total of 353 DEGs including 117 upregulated and 236 downregulated genes in CRC were identified based on GSE32323 data set. These DEGs were significantly enriched in the biological process related to the regulation of cell proliferation and 50 signaling pathways, such as "TGF-beta signaling pathway," "Wnt signaling pathway," and "Jak-STAT signaling pathway." GCG, ADH1B, SLC4A4, ZG16, and CLCA4 were the top five downregulated in CRC. FOXQ1, LGR5, CLDN1, KRT23, and DPEP1 were the top five upregulated in CRC. KRT23 expression could affect tumor stage and regional lymph node metastasis in CRC patients. FOXQ1 expression could affect tumor distant metastasis in CRC patients. Survival analysis indicated that SLC4A4 expression was associated with the prognosis of CRC patients. Prognostic prediction model developed based on age, tumor stage, and SLC4A4 expression exhibited an efficient performance in predicting 1-, 3-, and 5-year overall survival of CRC patients. In conclusion, the current study identified several genes and pathways related to CRC, which provided new insight in understanding molecular mechanism of tumorigenesis and development of CRC.

Keywords: colorectal cancer, gene, pathway, prognosis

\section{Introduction}

C OLORECTAL CANCER (CRC) is a major public health issue worldwide. There are over 1.8 million new CRC cases and 881,000 deaths in 2018 (Bray et al., 2018). Although researchers have made great efforts to reveal molecular mechanism of tumorigenesis and development of $\mathrm{CRC}$, it has not been completely clarified. Therefore, it is necessary to further explore CRC-related genes and pathways, which helps not only to understand molecular mechanism of the occurrence and development of CRC, but also to guide the development of promising diagnostic and prognostic biomarkers and optimal therapeutic strategies.

Over the past decade, microarray and high throughput sequencing technologies have been widely used in the biomedical field and have greatly promoted the development of diagnostic and prognostic biomarkers and therapeutic targets for cancer. Meanwhile, a large number of cancer- related data are stored in publicly available databases, such as The Cancer Genome Atlas (TCGA) and Gene Expression Omnibus (GEO). A reanalysis of these data could facilitate the discovery of a vast range of valuable biological information (Gao et al., 2018, 2019; Liu et al., 2019; Zhou et al., 2019). For instance, several novel genes associated with poor prognosis of patients with pancreatic ductal adenocarcinoma (PDAC) were identified by analyzing the array data from GEO. These genes might serve as potential diagnostic and prognostic biomarkers for PDAC (Zhou et al., 2019). Liu et al. (2019) revealed a novel robust four-gene metabolic signature predicting overall survival for hepatocellular carcinoma (HCC) by mining mRNA expression profiles and clinicopathological data from TCGA and GSE14520 data sets. The signature would be very useful for reflecting the dysregulated metabolic microenvironment and providing potential biomarkers for metabolic therapy and treatment response prediction in HCC (Liu et al., 2019). In 2012,

${ }^{1}$ School of Pharmacy, Yancheng Teachers' University, Yancheng, China.

${ }^{2}$ Department of Microbiology and Immunology, Shanxi Medical University, Tai yuan, China. 

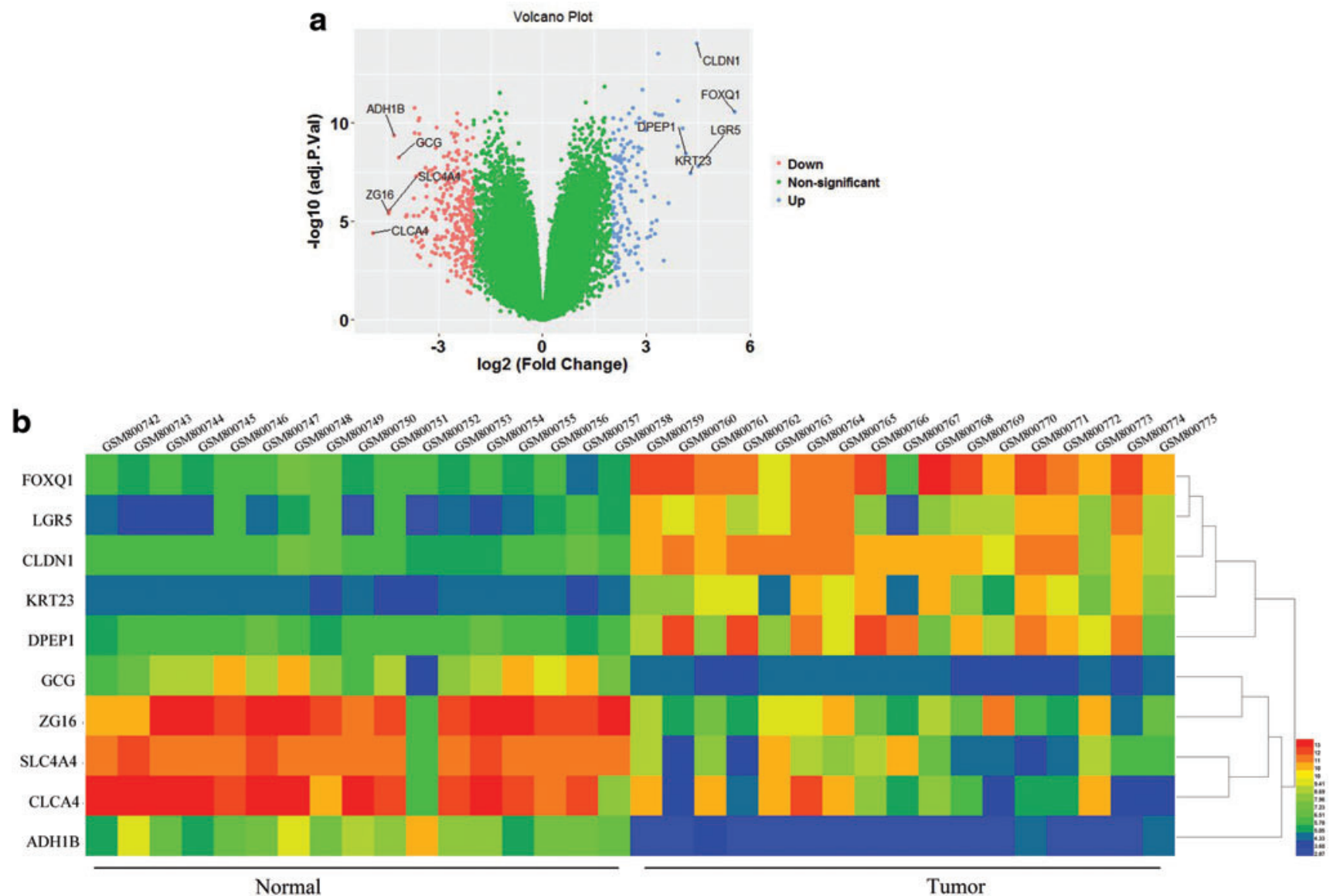

FIG. 1. Volcano plot and heatmap showing differentially expressed genes in CRC. (a) Volcano plot and (b) heatmap showing the expression levels of top five upregulated and downregulated genes. CRC, colorectal cancer. Color images are available online.

researchers comprehensively analyzed molecular characterization of human colon and rectal cancer based on TCGA data, and found that colon and rectum cancers had considerably similar patterns of genomic alteration (Cancer Genome Atlas Network, 2012). Twenty-four genes, such as ARID1A, SOX9, and FAM123B, were significantly mutated (Cancer Genome Atlas Network, 2012). In 2015, Guinney et al. (2015) developed a novel classification framework for predicting consensus molecular subtypes of CRC using aggregated gene expression data.

In this study, we reanalyzed gene expression profiles from GEO and TCGA databases and identified several genes and pathways related to CRC based on bioinformatics methods. In addition, we also developed a clinical model for predicting 1-, 3-, and 5-year overall survival of CRC patients.

\section{Materials and Methods}

\section{Gene expression profiles}

The RMA normalized gene expression profiles of cancerous and non-cancerous tissues from $17 \mathrm{CRC}$ patients were acquired from the GEO. These gene expression profiles were contributed by Khamas et al. (2012) and generated by Affymetrix HG-U133 Plus 2.0 arrays. In addition, the FPKM

Table 1. Gene Ontology Enrichment Analysis of the Differentially Expressed Genes

\begin{tabular}{llr}
\hline Categories & \multicolumn{1}{c}{ Terms } & $F D R$ \\
\hline GOTERM_BP_DIRECT & GO:0008285 $\sim$ negative regulation of cell proliferation & 0.004 \\
GOTERM_CC_DIRECT & GO:0005615 $\sim$ extracellular space & $<0.001$ \\
GOTERM_CC_DIRECT & GO:0005578 $\sim$ proteinaceous extracellular matrix & $<0.001$ \\
GOTERM_CC_DIRECT & GO:0070062 $\sim$ extracellular exosome & 0.001 \\
GOTERM_CC_DIRECT & GO:0005576 $\sim$ extracellular region & 0.001 \\
GOTERM_CC_DIRECT & GO:0005887 $\sim$ integral component of plasma membrane & 0.009 \\
GOTERM_CC_DIRECT & GO:0016324 $\sim$ apical plasma membrane & 0.011 \\
GOTERM_MF_DIRECT & GO:0045236 $\sim$ CXCR chemokine receptor binding & 0.019 \\
GOTERM_MF_DIRECT & GO:0008009 $\sim$ chemokine activity & 0.044 \\
\hline
\end{tabular}

BP, biological process; CC, cellular component; FDR, false discovery rate; GO, Gene Ontology; MF, molecular function. 
normalized gene expression profiles of 568 CRC tissues and 44 normal tissues and clinicopathological data from CRC patients were downloaded from TCGA database and processed by the Perl language.

\section{Identification of differentially expressed genes}

GEO2R tool was used to identify differentially expressed genes (DEGs) between cancerous and non-cancerous tissues from 17 CRC patients in GSE32323 data set. Genes with $|\log 2 \mathrm{FC}|>2$ and adjusted $p$-value $<0.05$ were considered as DEGs. According to $\log 2 \mathrm{FC}$ value, top five upregulated and downregulated genes were selected for further analysis. A volcano plot of DEGs was constructed by using ggplot2 and ggrepel packages. Furthermore, a heatmap of genes expression was also constructed by using HemI toolkit (Deng et al., 2014).

\section{Enrichment analysis of DEGs}

To understand the biological significance of DEGs, the Database for Annotation, Visualization, and Integrated Discovery (DAVID) v6.8) (Huang et al., 2009, 2019) and KOBAS 3.0 (Wu et al., 2006; Xie et al., 2011; Ai and Kong, 2018) online tools were used for enrichment analysis. There into DAVID was used to reveal Gene Ontology (GO) enriched by DEGs. KOBAS 3.0 was used to reveal KEGG pathways enriched by DEGs. GO terms with the false discovery rate $<0.05$ and KEGG pathways with the corrected $p$-value $<0.05$ were considered to be significantly enriched by DEGs.

\section{Validation and analysis of gene expression levels in $C R C$}

Wilcoxon test was used to validate the expression levels of top five upregulated and downregulated genes based on TCGA data sets. In addition, the association between expression levels of top five upregulated and downregulated genes and microsatellite instability (MSI) status of CRC patients was assessed by Wilcoxon test. $p$-value $<0.05$ was considered significant.

\section{The relationship between gene expression and clinicopathological characteristics}

Logistic regression analysis was used to explore the relationship between the expression levels of top five upregulated and downregulated genes and clinicopathological characteristics of CRC patients. The univariate cox regression analysis was used to identify factors associated with the overall survival of CRC patients. $p$-value $<0.05$ was considered significant.

\section{Development and evaluation of a prognostic prediction model for CRC patients}

A prognostic prediction model for CRC patients was developed based on potential prognostic factors and displayed in a nomogram. The concordance index (C-index) and receiver operating characteristic (ROC) curve were used to evaluate the performance of the prognostic prediction model. The risk score of each CRC patient was calculated based on the model. All CRC patients were divided into high- and
Table 2. KeGG Pathway Enrichment Analysis of the Differentially EXPRESSED Genes

\begin{tabular}{|c|c|c|}
\hline IDs & Terms & $\begin{array}{c}\text { Corrected } \\
\text { p-value }\end{array}$ \\
\hline hsa04060 & $\begin{array}{l}\text { Cytokine-cytokine receptor } \\
\text { interaction }\end{array}$ & $5.98 \mathrm{E}-07$ \\
\hline hsa04978 & Mineral absorption & $1.11 \mathrm{E}-05$ \\
\hline hsa00910 & Nitrogen metabolism & $1.46 \mathrm{E}-05$ \\
\hline hsa04972 & Pancreatic secretion & $4.30 \mathrm{E}-05$ \\
\hline hsa04610 & $\begin{array}{l}\text { Complement and coagulation } \\
\text { cascades }\end{array}$ & $1.12 \mathrm{E}-04$ \\
\hline hsa04960 & $\begin{array}{l}\text { Aldosterone-regulated sodium } \\
\text { reabsorption }\end{array}$ & $3.64 \mathrm{E}-04$ \\
\hline hsa05146 & Amoebiasis & $3.94 \mathrm{E}-04$ \\
\hline hsa05202 & $\begin{array}{l}\text { Transcriptional misregulation } \\
\text { in cancer }\end{array}$ & $4.06 \mathrm{E}-\mathrm{C}$ \\
\hline hsa04976 & Bile secretion & $4.99 \mathrm{E}-\mathrm{C}$ \\
\hline hsa04062 & Chemokine signaling pathway & $5.13 \mathrm{E}-\mathrm{C}$ \\
\hline hsa04151 & PI3K-Akt signaling pathway & $6.07 \mathrm{E}-($ \\
\hline hsa04964 & Proximal tubule bicarbonate & $6.81 \mathrm{E}-04$ \\
\hline
\end{tabular}
reclamation

hsa04350 TGF-beta signaling pathway

hsa01100

hsa05323

hsa05200

hsa04924

Metabolic pathways

Rheumatoid arthritis

Pathways in cancer

Renin secretion

Signaling pathways regulating pluripotency of stem cells

hsa04510

hsa03320

Focal adhesion

hsa05219

PPAR signaling pathway

Bladder cancer

Thyroid hormone signaling pathway

hsa04270

Vascular smooth muscle contraction

hsa04512

ECM-receptor interaction

hsa05144 Malaria

hsa04068

hsa04530

hsa04310

hsa00561

hsa05205

hsa00830

hsa04022

hsa04670

FoxO signaling pathway

Tight junction

Wnt signaling pathway

Glycerolipid metabolism

Proteoglycans in cancer

Retinol metabolism

cGMP-PKG signaling pathway

Leukocyte transendothelial migration

hsa04971

Gastric acid secretion

hsa05160 Hepatitis C

hsa00071

hsa04640

Fatty acid degradation

hsa04540

Hematopoietic cell lineage

Gap junction

hsa04974 Protein digestion and absorption

hsa01212

Fatty acid metabolism

Cell adhesion molecules (CAMs)

hsa04015

hsa04390

hsa04933

Rap1 signaling pathway

Hippo signaling pathway

AGE-RAGE signaling pathway in diabetic complications

hsa05217

Basal cell carcinoma

hsa05134

Legionellosis

hsa04630

hsa05150

Jak-STAT signaling pathway

hsa00500

Staphylococcus aureus infection

Starch and sucrose metabolism

Steroid hormone biosynthesis
$1.04 \mathrm{E}-03$

$1.07 \mathrm{E}-03$

$1.45 \mathrm{E}-03$

$1.87 \mathrm{E}-03$

$2.24 \mathrm{E}-03$

$2.25 \mathrm{E}-03$

$3.33 \mathrm{E}-03$

$3.38 \mathrm{E}-03$

$3.68 \mathrm{E}-03$

$4.35 \mathrm{E}-03$

$4.68 \mathrm{E}-03$

$5.33 \mathrm{E}-03$

$6.10 \mathrm{E}-03$

$7.27 \mathrm{E}-03$

$8.28 \mathrm{E}-03$

$9.20 \mathrm{E}-03$

$9.84 \mathrm{E}-03$

$1.14 \mathrm{E}-02$

$1.29 \mathrm{E}-02$

$1.63 \mathrm{E}-02$

$1.74 \mathrm{E}-02$

$1.82 \mathrm{E}-02$

$2.54 \mathrm{E}-02$

$2.70 \mathrm{E}-02$

$2.85 \mathrm{E}-02$

$2.85 \mathrm{E}-02$

$3.03 \mathrm{E}-02$

$3.18 \mathrm{E}-02$

3.32E-02

$3.75 \mathrm{E}-02$

$3.91 \mathrm{E}-02$

$4.05 \mathrm{E}-02$

$4.20 \mathrm{E}-02$

$4.20 \mathrm{E}-02$

$4.23 \mathrm{E}-02$

$4.50 \mathrm{E}-02$

$4.50 \mathrm{E}-02$

$4.66 \mathrm{E}-02$ 

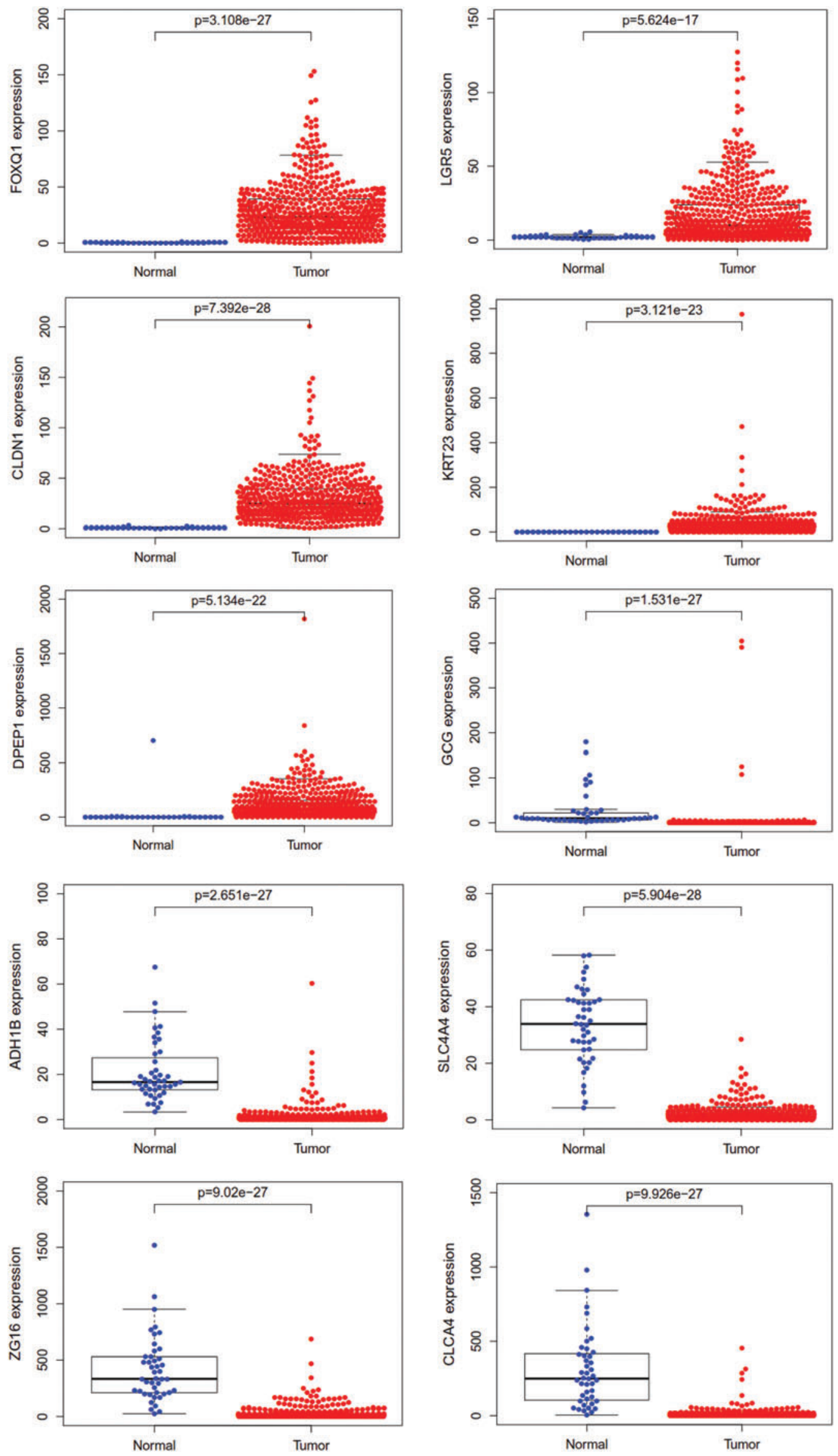

FIG. 2. The expression levels of top five upregulated and downregulated genes in CRC identified based on TCGA data sets. TCGA, The Cancer Genome Atlas. Color images are available online. 
Table 3. The Effect of the Expression Levels of Genes on Clinicopathological Characteristics of Colorectal Cancer

\begin{tabular}{|c|c|c|c|c|c|c|c|c|c|c|c|c|}
\hline \multirow[b]{3}{*}{ Gene } & \multicolumn{3}{|c|}{ Stage $(I+I I, I I I+I V)$} & \multicolumn{3}{|c|}{$T(T 1+T 2, T 3+T 4)$} & \multicolumn{3}{|c|}{$N(N 0, N 1)$} & \multicolumn{3}{|c|}{$M(M 0, M 1)$} \\
\hline & \multicolumn{3}{|c|}{$\mathrm{n}=526$} & \multicolumn{3}{|c|}{$\mathrm{n}=540$} & \multicolumn{3}{|c|}{$\mathrm{n}=540$} & \multicolumn{3}{|c|}{$\mathrm{n}=478$} \\
\hline & $\mathrm{p}$ & $O R$ & $95 \% C I$ & $\mathrm{p}$ & $O R$ & $95 \% C I$ & $\mathrm{p}$ & $O R$ & $95 \% C I$ & $\mathrm{p}$ & $O R$ & $95 \% C I$ \\
\hline$A D H 1 B$ & 0.067 & 1.073 & $0.995-1.156$ & 0.079 & 1.185 & $0.980-1.432$ & 0.069 & 1.064 & $0.995-1.138$ & 0.283 & 1.044 & 0.965 \\
\hline ZG16 & 0.660 & 0.999 & $0.996-1.003$ & 0.647 & 1.001 & $0.997-1.0$ & 0.6 & 0.999 & 0.996 & & 0.990 & 1.000 \\
\hline CLCA4 & 0.381 & 1.003 & $0.996-1.009$ & 0.530 & 0.998 & $0.992-1.0($ & 0.9 & 1.000 & $0.995-1.0$ & & 0.998 & 0.986 \\
\hline SLC $4 A 4$ & 0.130 & 0.943 & $0.874-1.017$ & 0.898 & 0.995 & $0.919-1.0^{\prime}$ & 0.0 & 0.932 & $0.863-1.0$ & & 0.856 & 0.728 \\
\hline$C L D N 1$ & 0.584 & & & 0.404 & 0.9 & & & 1.001 & & & 1.004 & \\
\hline$L G R 5$ & 0.604 & 1.002 & $0.993-1.011$ & 0.584 & 1.003 & 0.99 & 0.884 & 1.001 & 0.99 & 0.2 & 1.007 & 0.99 \\
\hline KRT23 & $\mathbf{0 . 0 3 0}$ & 1.005 & $1.000-1.009$ & 0.547 & 1.001 & $0.997-1.006$ & 0.036 & 1.004 & $1.000-1.008$ & 0.275 & 1.002 & $0.999-1.005$ \\
\hline$F O X Q 1$ & 0.063 & 1.007 & $1.000-1.015$ & 0.963 & 1.000 & $0.991-1.009$ & 0.248 & 1.004 & $0.997-1.012$ & $<0.001$ & 1.022 & $1.013-1.032$ \\
\hline$D P E P 1$ & 0.263 & 1.001 & $0.999-1.002$ & 0.471 & 0.999 & $0.998-1.001$ & 0.376 & 1.001 & 0.999-1.002 & 0.172 & 1.001 & $1.000-1.003$ \\
\hline$G C G$ & 0.507 & 1.036 & $0.933-1.152$ & 0.506 & 1.090 & $0.846-1.404$ & 0.507 & 1.038 & $0.929-1.160$ & 0.249 & 1.034 & 0.977-1.094 \\
\hline
\end{tabular}

Bold values indicate significant effects.

CI, confidence interval; OR, odds ratio.

low-risk group based on median risk score. Kaplan-Meier survival curves and a scatter diagram were used to show prognostic difference between two groups. These analyses were performed by survival, rms, survcomp, and timeROC packages in $\mathrm{R}$ software.

\section{Results}

\section{Identification and analysis of DEGs}

A total of 117 upregulated and 236 downregulated genes in CRC were identified based on GSE32323 data set. FOXQ1, LGR5, CLDN1, KRT23, and DPEP1 were top five up-regulated genes and $G C G, A D H 1 B, S L C 4 A 4, Z G 16$, and $C L C A 4$ were top five down-regulated genes (Fig. 1). The functional enrichment analysis showed that DEGs were significantly enriched in the following nine GO terms: GO:0008285 negative regulation of cell proliferation, GO:0005615 extracellular space, GO:0005578 proteinaceous extracellular matrix, GO:0070062 extracellular exosome, GO:0005576 extracellular region, GO:0005887 integral component of plasma membrane, GO:0016324 apical plasma membrane, GO:0045236 CXCR chemokine receptor binding, and GO:0008009 chemokine activity (Table 1). The enrichment analysis based on KEGG pathway showed that DEGs were significantly enriched in 50 pathways (Table 2). There into hsa04060 cytokine-cytokine receptor interaction, hsa04978 mineral absorption, hsa00910 nitrogen metabolism, hsa04972 pancreatic secretion, and hsa04610 complement and coagulation cascades were top five pathways significantly enriched by DEGs (Table 2).

\section{Validation and analysis of gene expression levels in $C R C$}

TCGA data sets showed that the expression levels of FOXQ1, LGR5, CLDN1, KRT23, and DPEP1 were upregulated in CRC tissues, and the expression levels of $G C G$, $A D H 1 B, S L C 4 A 4, Z G 16$, and CLCA4 were downregulated in CRC tissues, which were consistent with the results of GSE32323 data set (Fig. 2). In addition, logistic regression analysis indicated that KRT23 expression in CRC tissues could affect tumor stage $(\mathrm{OR}=1.005$, 95\% $\mathrm{CI}=1.000-$ $1.009, p=0.03)$ and regional lymph node metastasis $(\mathrm{OR}=$ $1.004,95 \% \mathrm{CI}=1.000-1.008, p=0.036$ ) (Table 3). FOXQ1 expression in CRC tissues could affect tumor distant metastasis $(\mathrm{OR}=1.022,95 \% \quad \mathrm{CI}=1.013-1.032, \quad p<0.001)$ (Table 3). The univariate cox regression analysis showed that SLC4A4 expression was correlated with the overall survival of $\mathrm{CRC}$ patients $(\mathrm{HR}=0.872,95 \% \mathrm{CI}=0.762-$ 0.999, $p=0.048$ ) (Table 4). After adjusting for $B R A F$ mutation status, SLC4A4 expression still could affect the overall survival of $\mathrm{CRC}$ patients $(\mathrm{HR}=0.87,95 \% \mathrm{CI}=0.75-$ $0.99, p=0.041)$.

\section{Development and evaluation of a prognostic prediction model for CRC patients}

Age, tumor stage, and SLC4A4 expression were used to develop a prognostic prediction model (Fig. 3a). The C-index was 0.737 for the prognostic prediction model, suggesting that the model had moderate discrimination ability. The area under the ROC curve for 1-, 3-, and 5-year

Table 4. Identification of Factors Associated with the Overall Survival of Colorectal Cancer Patients

\begin{tabular}{lccr}
\hline Characteristics & $H R$ & $95 \%$ CI & \multicolumn{1}{c}{$\mathrm{p}$} \\
\hline Age & $\mathbf{1 . 0 3 6}$ & $\mathbf{1 . 0 1 6}-\mathbf{1 . 0 5 6}$ & $<\mathbf{0 . 0 0 1}$ \\
Gender & 1.133 & $0.751-1.709$ & 0.552 \\
Stage & $\mathbf{2 . 1 0 3}$ & $\mathbf{1 . 6 6 8 - 2 . 6 5 3}$ & $<\mathbf{0 . 0 0 1}$ \\
ADH1B & 1.029 & $0.967-1.096$ & 0.360 \\
ZG16 & 0.994 & $0.986-1.001$ & 0.111 \\
CLCA4 & 1.000 & $0.992-1.008$ & 0.994 \\
SLC4A4 & $\mathbf{0 . 8 7 2}$ & $\mathbf{0 . 7 6 2 - 0 . 9 9 9}$ & $\mathbf{0 . 0 4 8}$ \\
CLDN1 & 1.002 & $0.993-1.012$ & 0.611 \\
LGR5 & 1.002 & $0.992-1.012$ & 0.715 \\
KRT23 & 0.997 & $0.991-1.002$ & 0.253 \\
FOXQ1 & 1.005 & $0.997-1.013$ & 0.252 \\
DPEP1 & 1.000 & $0.998-1.002$ & 0.867 \\
GCG & 1.001 & $0.992-1.011$ & 0.800 \\
\hline
\end{tabular}

Bold values indicate significant associations.

CI, confidence interval; HR, hazard ratio. 


\section{a}

Points

age

stage

SLC4A4

Total Points

1-Year Overall Survival

3-Year Overall Survival

5-Year Overall survival
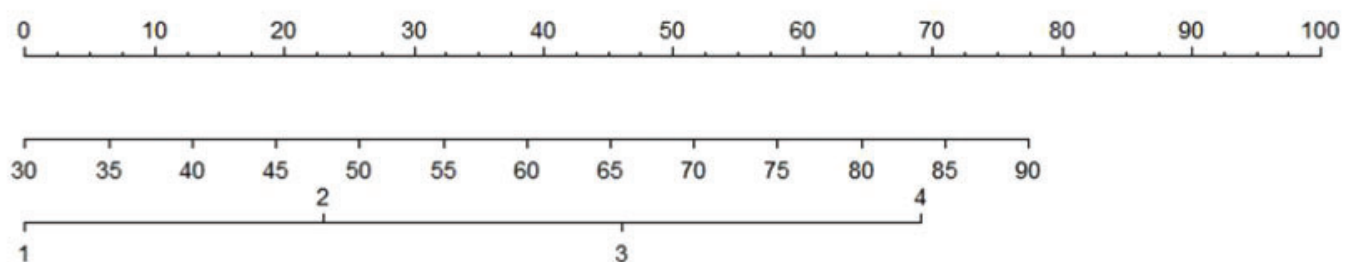

\begin{tabular}{rllllll}
\hline 30 & 25 & 20 & 15 & 10 & 5 & 0
\end{tabular}

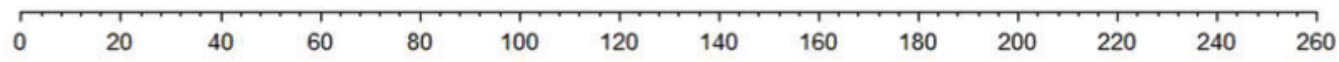
10

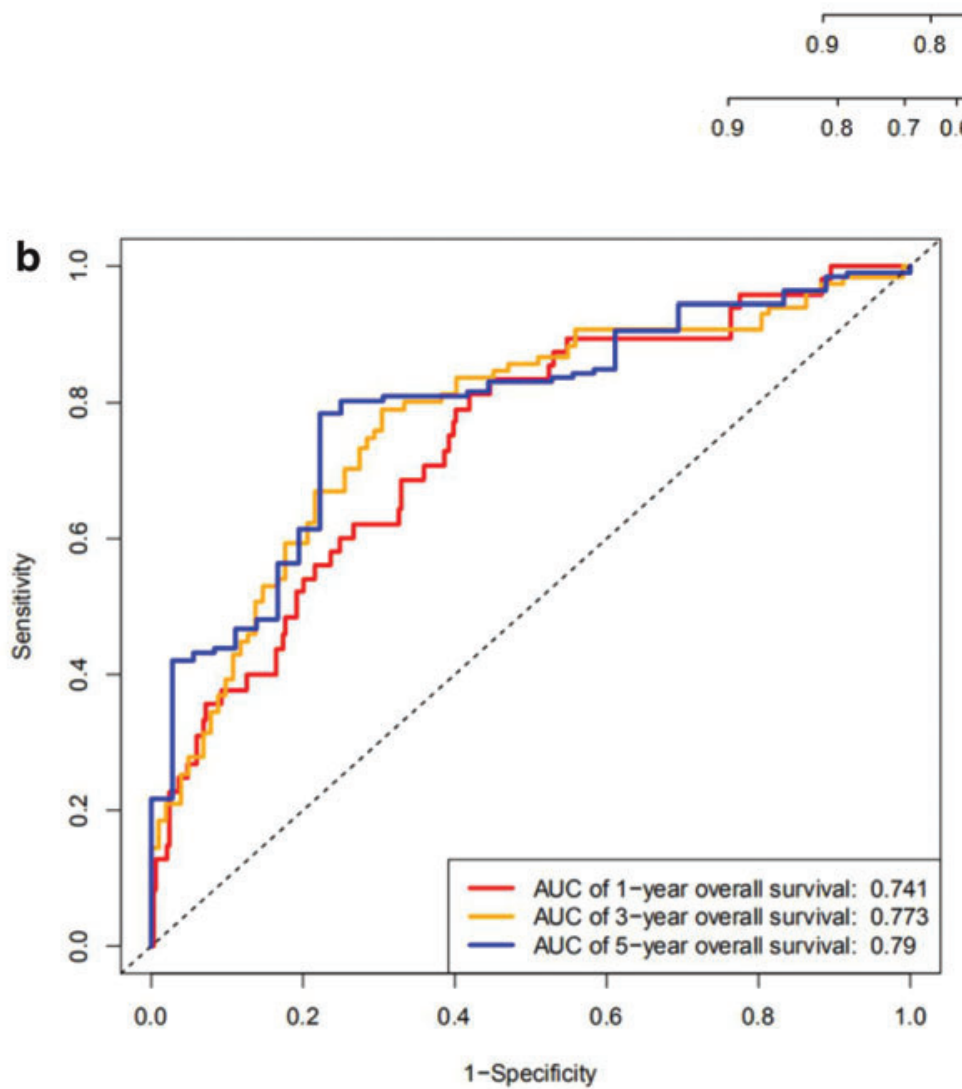

FIG. 3. The nomogram and ROC curve analysis of prognostic prediction model for CRC patients. (a) Nomogram and (b) ROC curve. ROC, receiver operating characteristic. Color images are available online.

overall survival were $0.741,0.773$, and 0.790 , respectively, suggesting that the prognostic prediction model had a moderate sensitivity and specificity (Fig. 3b). In addition, Kaplan-Meier plot showed that the overall survival of the high-risk group was significantly poorer than that of the low-risk group ( $p=2.121 \mathrm{e}-12$, Fig. 4a). The scatter diagram showed that mortality of the high-risk group was significantly higher than that of the low-risk group (29\% vs. $6.5 \%$, $p<0.001$, Fig. 4b).

\section{Discussion}

The incidence and mortality of CRC were still on the rise in many countries. Identification of genes and pathways related to CRC would be helpful for the diagnosis and treatment of the disease. In the current study, we identified 353 CRC-related genes by mining gene expression profiles of GSE32323 data set. These genes were significantly enriched in cancer-related GO terms and signaling pathways. For instance, PI3K-Akt pathway was one of the most frequently activated signal transduction pathways in human cancer, and strongly implicated in cancer pathogenesis (Guo et al., 2015; Chen et al., 2016a; Wang et al., 2019). TGFbeta signaling pathway could promote tumor growth, invasion, and metastasis through inducing angiogenesis, epithelial-mesenchymal transition, and immune escape (Roberts and Wakefield, 2003). Targeting the effectors of these pathways might be a promising therapeutic approach. 
a

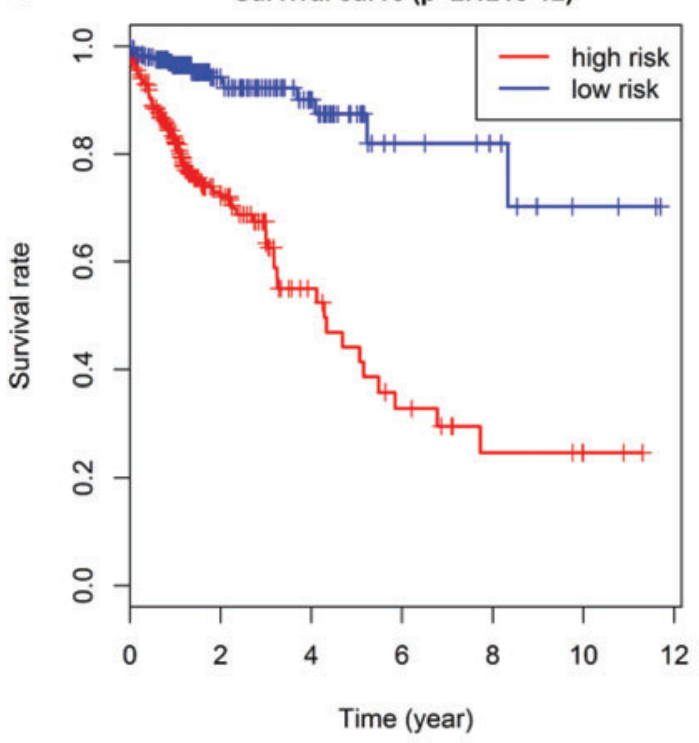

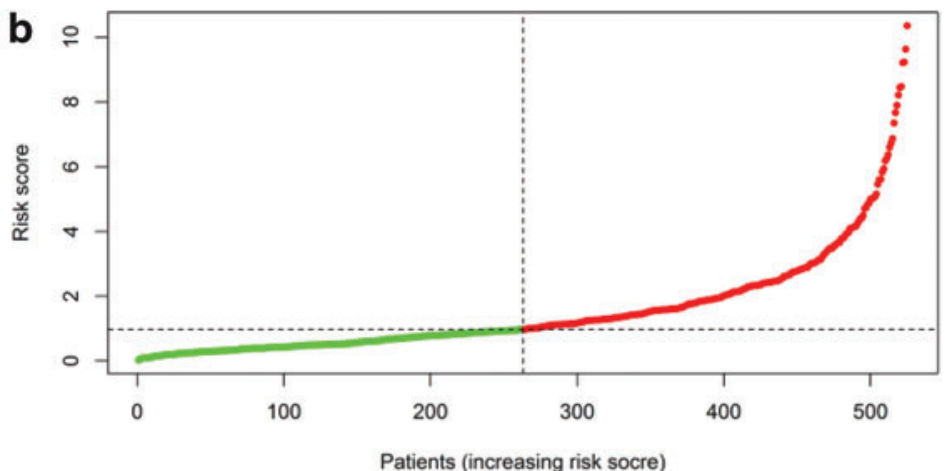

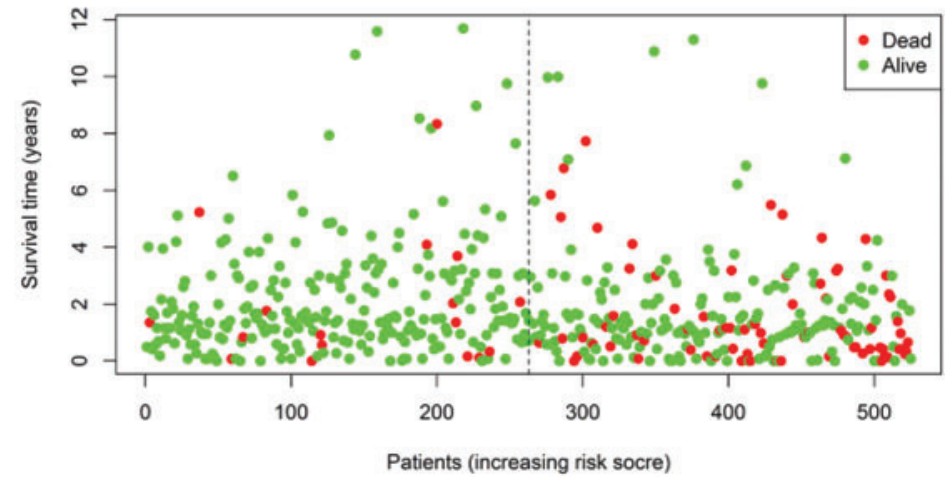

FIG. 4. Survival analysis of CRC patients in the high- and low-risk group. (a) Survival curve of CRC patients in the highand low-risk group and (b) scatter diagram for risk score, survival time, and status of CRC patients. Color images are available online.

Top five upregulated (FOXQ1, LGR5, CLDN1, KRT23, and $D P E P 1)$ and downregulated (GCG, ADH1B, SLC4A4, ZG16, and CLCA4) genes in CRC tissues were further analyzed based on TCGA data sets. The expression levels of FOXQ1, LGR5, CLDN1, KRT23, DPEP1, GCG, SLC4A4, and $C L C A 4$ were associated with MSI status of CRC patients (Fig. 5). The expression levels of FOXQ1, LGR5, CLDN1, KRT23, and DPEP1 in CRC patients with MSI were significantly lower than those in CRC patients without MSI. The expression levels of GCG,SLC4A4, and CLCA4 in CRC patients with MSI were significantly higher than those in CRC patients without MSI. FOXQ1 was a member of the forkhead box protein family, which was characterized by a conserved 110-amino acid DNA-binding motif called the forkhead or winged helix domain. It was involved in many important biological processes, such as embryonic development, metabolism, and cell cycle regulation (Zhu, 2016). In addition, FOXQ1 had been recognized as an oncogene that was overexpressed in a variety of cancers, such as CRC, liver cancer, and esophageal cancer (Kaneda et al., 2010; Wang et al., 2013; Pei et al., 2015). The current research found that high expression of FOXQ1 in CRC tissues could increase the risk of tumor distant metastasis. LGR5 was an established cancer stem cell marker and could promote the proliferation of CRC cells via the $\mathrm{Wnt} / \beta$-catenin signaling pathway, which was a critical pathway in the process of tumor initiation and growth (Lin et al., 2015). CLDN1, a member of the claudin family, was an integral membrane protein and a component of tight junction strands. It had been found abnormally expressed in a variety of tumors, such as cervical cancer, oral squamous cell carcinoma, and CRC (Tang et al., 2011; Sappayatosok and Phattar- ataratip, 2015; Zhang et al., 2016). In human CRC tissues, CLDN1 expression was upregulated (Tang et al., 2011). Furthermore, it could regulate cellular transformation and metastatic behavior in colon cancer (Dhawan et al., 2005). KRT23 was a member of the keratin family that was an intermediate filament protein responsible for the structural integrity of epithelial cells. KRT23 could act as an important cellular factor that promoted CRC growth by activating the expression of human telomerase reverse transcriptase (Zhang et al., 2017). The current research indicated that high expression of KRT23 in CRC tissues could increase the risk of advanced tumor stages and regional lymph node metastasis. DPEP1 was a zinc-dependent metalloproteinase involved in the metabolism of glutathione and other similar proteins by dipeptide hydrolysis. It was initially considered as a tumor suppressor gene in PDAC and breast cancer (Green et al., 2009; Zhang et al., 2012). On the contrary, several studies demonstrated that DPEP1 was highly expressed in $\mathrm{CRC}$ and promoted metastasis by regulating expression level of E-cadherin in colon cancer (Toiyama et al., 2011; Park et al., 2016). GCG was a preproprotein that was cleaved into four distinct mature peptides. One of these, glucagon, was a ligand for a specific G-protein linked receptor whose signaling pathway controlled cell proliferation. The current results showed that GCG expression was significantly downregulated in CRC tissues. Thus, we inferred that GCG might play a major role in suppressing the development of CRC. ADH1B was a member of the alcohol dehydrogenase family that metabolized a wide variety of substrates, such as ethanol, hydroxysteroids, and lipid peroxidation products. Alcohol carcinogenesis mostly affected tissues directly exposed to alcohol ingestion, such as 

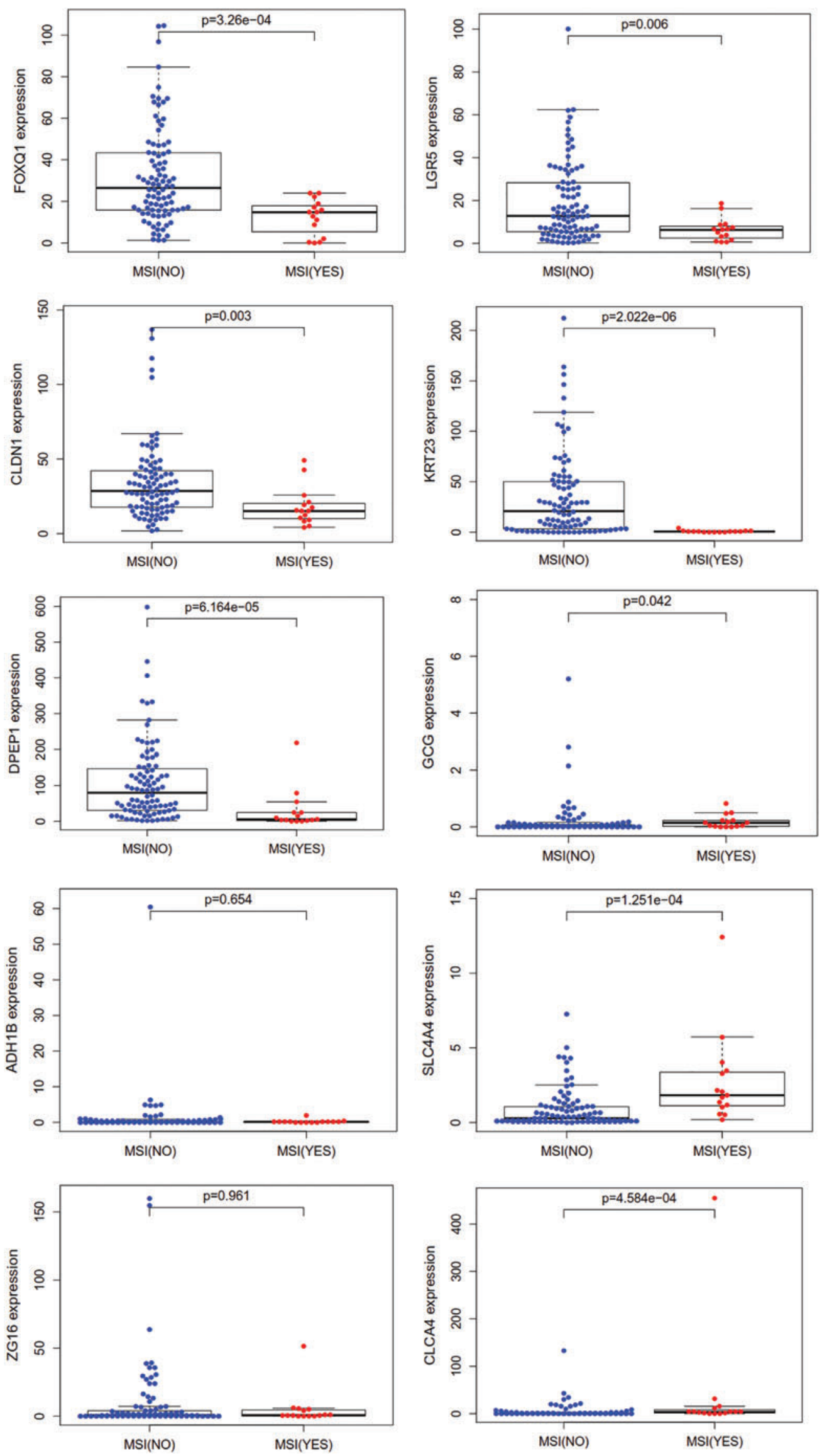

FIG. 5. The association between expression levels of top five upregulated and downregulated genes and MSI status of CRC patients (15 CRC patients with MSI and 92 CRC patients without MSI). MSI, microsatellite instability. Color images are available online. 
esophagus, larynx and colon. Although the molecular function of ADH1B in CRC was not directly reported, genetic polymorphism (rs1229984) in $A D H 1 B$ gene was revealed to be associated with CRC risk (Crous-Bou et al., 2013; Chen et al., 2014), suggesting that $A D H 1 B$ might be an important genetic susceptibility gene for CRC. SLC4A4 was a sodium bicarbonate cotransporter responsible for the regulation of bicarbonate secretion and absorption and intracellular $\mathrm{pH}$. Xiao et al. (2019) found that miR-223-3p could enhance cell proliferation and metastasis by downregulating SLC4A4 in clear cell renal cell carcinoma, indicating that SLC4A4 might serve as a tumor suppressor gene. We found that SLC4A4 expression was downregulated in CRC tissues, and high expression of SLC4A4 could reduce the risk of death in CRC patients. ZG16 was a secreted protein downregulated in liver cancer and CRC (Zhou et al., 2007). Chen et al. (2016b) demonstrated that loss of ZG16 was helpful for stemness and progression of CRC, which might provide a promising therapeutic strategy for advanced CRC. CLCA4 belonged to the calcium sensitive chloride conductance protein family and could act as a tumor suppressor in tumorigenesis and progression of several human cancers, including HCC, CRC, and bladder cancer (Hou et al., 2017; Liu et al., 2018; Chen et al., 2019).

Recently, the development of a prognostic prediction model has become a research hotspot. In this study, we observed that age, tumor stage, and SLC4A4 expression were associated with overall survival of CRC patients. Subsequently, a model for predicting prognosis of CRC patients was developed base on these three prognostic factors. The model had a good performance in predicting 1-, 3-, and 5year overall survival of CRC patients, which would help clinicians make better clinical decisions and follow-up surveillance. However, due to limitations of research, systemic therapies and other known prognostic factors were not included in survival analysis. Thus the prognosis model needed to be further improved.

\section{Conclusions}

This study identified several genes and pathways related to $\mathrm{CRC}$, which provided not only new diagnostic markers and therapeutic targets for CRC, but also a promising prognostic indicator for CRC patients.

\section{Authors' Contributions}

X.G., the first author and the corresponding author, put forward the ideas of this article, wrote the article, and analyzed the data. As a coauthor, J.Y. acquired the data and helped in revising the article.

\section{Disclosure Statement}

The authors declare no conflict of interest.

\section{Funding Information}

No funding was received for this article.

\section{References}

Ai, C., and Kong, L. (2018). CGPS: a machine learning-based approach integrating multiple gene set analysis tools for better prioritization of biologically relevant pathways. J Genet Genomics 45, 489-504.

Bray, F., Ferlay, J., Soerjomataram, I., Siegel, R.L., Torre, L.A., and Jemal, A. (2018). Global cancer statistics 2018: GLOBOCAN estimates of incidence and mortality worldwide for 36 cancers in 185 countries. CA Cancer J Clin 68, 394-424.

Cancer Genome Atlas Network. (2012). Comprehensive molecular characterization of human colon and rectal cancer. Nature 487, 330-337.

Chen, C., Wang, L., Liao, Q., Xu, L., Huang, Y., Zhang, C., et al. (2014). Association between six genetic polymorphisms and colorectal cancer: a meta-analysis. Genet Test Mol Biomarkers 18, 187-195.

Chen, H., Liu, Y., Jiang, C.J., Chen, Y.M., Li, H., and Liu, Q.A. (2019). Calcium-Activated Chloride Channel A4 (CLCA4) plays inhibitory roles in invasion and migration through suppressing epithelial-mesenchymal transition via PI3K/AKT signaling in colorectal cancer. Med Sci Monit 25, 4176-4185. Chen, H., Zhou, L., Wu, X., Li, R., Wen, J., Sha, J., et al. (2016a). The PI3K/AKT pathway in the pathogenesis of prostate cancer. Front Biosci (Landmark Ed) 21, 1084-1091.

Chen, X., Du, P., She, J., Cao, L.; Li, Y., and Xia, H. (2016b). Loss of ZG16 is regulated by miR-196a and contributes to stemness and progression of colorectal cancer. Oncotarget 7, 86695-86703.

Crous-Bou, M., Rennert, G., Cuadras, D., Salazar, R., Cordero, D., Saltz, Rennert, H., et al. (2013). Polymorphisms in alcohol metabolism genes ADH1B and ALDH2, alcohol consumption and colorectal cancer. PLoS One 8, e80158.

Deng, W., Wang, Y., Liu, Z., Cheng, H., and Xue, Y. (2014). HemI: a toolkit for illustrating heatmaps. PLoS One 9, e111988.

Dhawan, P., Singh, A.B., Deane, N.G., No, Y., Shiou, S.R., Schmidt, C., et al. (2005). Claudin-1 regulates cellular transformation and metastatic behavior in colon cancer. J Clin Invest 115, 1765-1776.

Gao, X., Wang, X., and Zhang, S. (2018). Bioinformatics identification of crucial genes and pathways associated with hepatocellular carcinoma. Biosci Rep 38, pii: BSR20181441.

Gao, X., Wang, J., and Zhang, S. (2019). Integrated bioinformatics analysis of hub enes and pathways in anaplastic thyroid carcinomas. Int $\mathbf{J}$ Endocrinol 2019, 9651380.

Green, A.R., Krivinskas, S., Young, P., Rakha, E.A., Paish, E.C., Powe, D.G., et al. (2009). Loss of expression of chromosome 16q genes DPEP1 and CTCF in lobular carcinoma in situ of the breast. Breast Cancer Res Treat 113, 59-66.

Guinney, J., Dienstmann, R., Wang, X., de Reyniès, A., Schlicker, A., Soneson, C., et al. (2015). The consensus molecular subtypes of colorectal cancer. Nat Med 21,1350 1356.

Guo, H., German, P., Bai, S., Barnes, S., Guo, W., Qi, X., et al. (2015). The PI3K/AKT Pathway and Renal Cell Carcinoma. J Genet Genomics 42, 343-353.

Hou, T., Zhou, L., Wang, L., Kazobinka, G., Zhang, X., and Chen, Z. (2017). CLCA4 inhibits bladder cancer cell proliferation, migration, and invasion by suppressing the PI3K/AKT pathway. Oncotarget 8, 93001-93013.

Huang, D.W., Sherman, B.T., and Lempicki, R.A. (2009). Bioinformatics enrichment tools: paths toward the comprehensive functional analysis of large gene lists. Nucleic Acids Res 37, 1-13.

Huang, D.W., Sherman, B.T., and Lempicki, R.A. (2019). Systematic and integrative analysis of large gene lists using DAVID Bioinformatics Resources. Nat Protoc 4, 44-57. 
Khamas, A., Ishikawa, T., Shimokawa, K., Mogushi, K., Iida, S., Ishiguro, M., et al. (2012). Screening for epigenetically masked genes in colorectal cancer Using 5-Aza-2'-deoxycytidine, microarray and gene expression profile. Cancer Genomics Proteomics 9, 67-75.

Kaneda, H., Arao, T., Tanaka, K., Tamura, D., Aomatsu, K., Kudo, K., et al. (2010). FOXQ1 is overexpressed in colorectal cancer and enhances tumorigenicity and tumor growth. Cancer Res 70, 2053-2063.

Lin, Y.U., Wu, T., Yao, Q., Zi, S., Cui, L., Yang, M., et al. (2015). LGR5 promotes the proliferation of colorectal cancer cells via the $\mathrm{Wnt} / \beta$-catenin signaling pathway. Oncol Lett 9, 2859-2863.

Liu, G.M., Xie, W.X., Zhang, C.Y., and Xu, J.W. (2019). Identification of a four-gene metabolic signature predicting overall survival for hepatocellular carcinoma. J Cell Physiol 235, 1624-1636.

Liu, Z., Chen, M., Xie, L.K., Liu, T., Zou, Z.W., Li, Y., et al. (2018). CLCA4 inhibits cell proliferation and invasion of hepatocellular carcinoma by suppressing epithelialmesenchymal transition via PI3K/AKT signaling. Aging (Albany NY) 10, 2570-2584.

Park, S.Y., Lee, S.J., Cho, H.J., Kim, T.W., Kim, J.T., Kim, J.W., et al. (2016). Dehydropeptidase 1 promotes metastasis through regulation of E-cadherin expression in colon cancer. Oncotarget 7, 9501-9512.

Pei, Y., Wang, P., Liu, H., He, F., and Ming, L. (2015). FOXQ1 promotes esophageal cancer proliferation and metastasis by negatively modulating CDH1. Biomed Pharmacother 74, 89-94.

Roberts, A.B., and Wakefield, L.M. (2003). The two faces of transforming growth factor beta in carcinogenesis. Proc Natl Acad Sci U S A 100, 8621-8623.

Sappayatosok, K., and Phattarataratip, E. (2015). Overexpression of claudin- 1 is associated with advanced clinical stage and invasive pathologic characteristics of oral squamous cell carcinoma. Head Neck Pathol 9, 173-180.

Tang, W., Dou, T., Zhong, M., and Wu, Z. (2011). Dysregulation of claudin family genes in colorectal cancer in a Chinese population. Biofactors 37, 65-73.

Toiyama, Y., Inoue, Y., Yasuda, H., Saigusa, S., Yokoe, T., Okugawa, Y., et al. (2011). DPEP1, expressed in the early stages of colon carcinogenesis, affects cancer cell invasiveness. J Gastroenterol 46, 153-163.

Wang, H., Yang, X., Guo, Y., Shui, L., Li, S., Bai, Y., et al. (2019). HERG1 promotes esophageal squamous cell carcinoma growth and metastasis through TXNDC5 by activating the PI3K/AKT pathway. J Exp Clin Cancer Res 38, 324.

Wang, W., He, S., Ji, J., Huang, J., Zhang, S., et al. (2013). The prognostic significance of FOXQ1 oncogene overexpression in human hepatocellular carcinoma. Pathol Res Pract 209, 353-358.

Wu, J., Mao, X., Cai, T., Luo, J., and Wei, L. (2006). KOBAS server: a web-based platform for automated annotation and pathway identification. Nucleic Acids Res 34, W720-W724.

Xiao, W., Wang, X., Wang, T., and Xing, J. (2019). MiR-223$3 \mathrm{p}$ promotes cell proliferation and metastasis by downregulating SLC4A4 in clear cell renal cell carcinoma. Aging (Albany NY) 11, 615-633.

Xie, C., Mao, X., Huang, J., Ding, Y., Wu, J., Dong, S., et al. (2011). KOBAS 2.0: a web server for annotation and identification of enriched pathways and diseases. Nucleic Acids Res 39, W316-W322.

Zhang, G., Schetter, A., He, P., Funamizu, N., Gaedcke, J., Ghadimi, B.M., et al. (2012). DPEP1 inhibits tumor cell invasiveness, enhances chemosensitivity and predicts clinical outcome in pancreatic ductal adenocarcinoma. PLoS One 7, e31507.

Zhang, N., Zhang, R., Zou, K., Yu, W., Guo, W., Gao, Y., et al. (2017). Keratin 23 promotes telomerase reverse transcriptase expression and human colorectal cancer growth. Cell Death Dis 8, e2961.

Zhang, W.N., Li, W., Wang, X.L., Hu, Z., Zhu, D., Ding, W.C., et al. (2016). CLDN1 expression in cervical cancer cells is related to tumor invasion and metastasis. Oncotarget 7, 87449-87461.

Zhou, J., Hui, X.L.,Mao, Y., and Fan, L.Y. (2019). Identification of novel genes associated with a poor prognosis in pancreatic ductal adenocarcinoma via a bioinformatics analysis. Biosci Rep 39, pii: BSR20190625.

Zhou, Y.B., Cao, J.B., Yang, H.M., Zhu, H., Xu, Z.G., Wang, K.S., et al. (2007). hZG16, a novel human secreted protein expressed in liver, was down-regulated in hepatocellular carcinoma. Biochem Biophys Res Commun 355, 679-686.

Zhu, H. (2016). Forkhead box transcription factors in embryonic heart development and congenital heart disease. Life Sci 144, 194-201.

Address correspondence to: Xueren Gao, PhD

School of Pharmacy

Yancheng Teachers' University Yancheng 224007

Jiangsu

China

E-mail: gxr871230@126.com

Received for publication September 9, 2019; received in revised form January 7, 2020; accepted January 8, 2020. 